\title{
Matriz de ações de alimentação e nutrição na Atenção Básica de Saúde: referencial para a formação do nutricionista no contexto da educação por competências
}

\author{
Matrix of food and nutrition actions in primary health care: \\ a benchmark for training of nutritionists in the context \\ of education by skillsets
}

Túlio da Silva Junqueira ${ }^{1}$

Rosângela Minardi Mitre Cotta ${ }^{1}$

${ }^{1}$ Departamento de Nutrição e Saúde, Universidade Federal de Viçosa. Av. Peter Henry Rolfs s/n, Campus Universitário. 36.570-000 Viçosa MG Brasil. tuliojunqueira@gmail.com

\begin{abstract}
This study had two objectives, namely to conduct a critical review of studies that underpin the training and practice of nutritionists in the context of Primary Health Care (PHC) in the Unified Health System (SUS), and evaluate the contribution of the matrix of food and nutrition actions in primary health care in this discussion. A protocol was developed to conduct the review in a systematic way. For the selection of studies, major databases were consulted, i.e. those used in health facilities, using intersections of the key search words: Nutritionist, National Policy on Food and Nutrition; National Curriculum Guidelines on health, Skill-based education; PHC. The array of actions can contribute to the training of nutritionists in the context of skill-based education to serve as a benchmark for actions, skills and abilities to train individuals, such that they are better able to cope with the needs of the population with emphasis on the SUS. Using it as a benchmark for training can contribute to the improvement of the latter and governmental actions based on the adoption of action on food and nutrition in PHC, in an effort complementing actions of all the other health programs, in particular the Family Health Strategy.
\end{abstract}

Key words Nutritionist, Primary Health Care, National curriculum guidelines on health, Professional training in health
Resumo O presente estudo teve duplo objetivo: realizar uma revisão crítica dos estudos que orientam a formação e a prática dos nutricionistas no contexto da Atenção Primária em Saúde (APS) no SUS, e avaliar a contribuição da Matriz de ações de alimentação e nutrição na atenção básica de saúde para esta discussão. Foi desenvolvido um protocolo para conduzir de forma sistematizada a revisão. Para a seleção dos estudos, foram consultadas as principais bases de dados em saúde onde se utilizou interseções dos conjuntos dos termos: Nutricionista, Politica Nacional de Alimentação e Nutrição; Diretrizes Curriculares Nacionais na saúde, Educação baseada em competências; APS. A matriz de ações pode contribuir para a formação do nutricionista dentro do contexto da educação por competências ao servir de referencial de açães, competências e habilidades por formar indivíduos mais capazes de lidar com as necessidades da população com ênfase no SUS. Ao utilizá-la como referencial de formação pode-se contribuir com o aperfeiçoamento desta e das ações governamentais a partir da adoção de ações de alimentação e nutrição na APS, num esforço convergente às demais iniciativas dos programas de saúde, em especial a Estratégia Saúde da Família. Palavras-chave Nutricionista, Atenção Primaria em Saúde, Diretrizes curriculares nacionais na saúde, Formação em saúde 


\section{Introdução}

No Brasil, a adequada formação profissional na área de saúde deve ter como referência o Sistema Único de Saúde (SUS). Neste contexto, as instituições de ensino superior, pautadas nas Diretrizes Curriculares Nacionais (DCN), devem construir e/ou reconstruírem seus projetos pedagógicos visando tanto a formação dos estudantes como a Educação Permanente dos profissionais em serviços, para atender as demandas e diretrizes do nosso sistema nacional de saúde - o SUS.

As DCN preveem mudanças curriculares que orientem uma formação profissional segundo as diretrizes e os princípios do Sistema Único de Saúde, considerando as necessidades de saúde individuais e coletivas e reorientando o modelo assistencial, de forma a valorizar a promoção da saúde, salientando os seus determinantes sociais ${ }^{1}$.

Desde esta perspectiva, o SUS foi concebido tendo como referencia a ideia de mudanças paradigmáticas: do paradigma biomédico/mecanicista, baseado no padrão reducionista, orientado para a doença, centrado no hospital e na especialização presente na tradicional lógica educacional das universidades e, por conseguinte na prestação dos serviços de saúde, para o paradigma da Produção Social da Saúde, com sua concepção holística e sistêmica do processo saúde-doençaadoecimento, orientado pela humanização, alteridade e trabalho em equipe interdisciplinar, cuja ênfase está na Atenção Primária à Saúde (APS) ${ }^{2-4}$. Assim, os estudantes, futuros profissionais nutricionistas, devem ser formados segundo os marcos das DCN para atuar no SUS 5 .

Destaca-se a necessidade de que se implementem mudanças paradigmáticas também nas universidades, orientando os currículos para a formação por competências. Segundo Lizarraga ${ }^{6}$ e Siqueira-Batista et al. ${ }^{1}$, a educação centrada em competências refere-se a uma aprendizagem baseada nos conhecimentos, atitudes e habilidades, necessária para que os estudantes e profissionais atuem de maneira ativa, responsável e criativa na construção de seu projeto de vida, tanto pessoal e social como profissional.

Em contrapartida, a evidência científica destaca que na prática os currículos dos cursos de nível superior em nutrição do Brasil se caracterizam pela ênfase no perfil profissional, objetivos e grade curricular, não apresentando, portanto, um projeto pedagógico fundamentado por princípios filosóficos e psicopedagógicos explícitos, necessários à definição das competências, dos objetivos de aprendizagem a serem alcançados, das estratégias educacionais e metodológicas e do sistema de avaliação da aprendizagem ${ }^{5,7-9}$.

Destarte, o mundo contemporâneo aponta para a necessidade de se repensar as práticas de formação dos nutricionistas. A legitimação dos órgãos oficiais, como o Ministério da Educação (MEC) e o Ministério da Saúde (MS), no que tange à mudança, já foi lançada. Contudo, as mudanças não ocorrem somente por meio de decretos, leis e diretrizes, é preciso também transformar pessoas já que os relacionamentos humanos fazem parte fundamental do processo de mudança ${ }^{10,11}$.

O ano de 2000 marca a implantação das $\mathrm{DCN}^{12}$. Para os cursos de graduação em Nutrição a referência é a Resolução no 5 CES/CNE, de 7 de novembro de 2001 do Conselho Nacional de Educação, que define princípios, fundamentos, condições e procedimentos da formação dos nutricionistas ${ }^{13}$.

Esta reorientação do processo de formação dos profissionais da saúde, em resposta às necessidades do sistema de saúde pública, deve ser pautada pela melhoria da qualidade e da resolubilidade da atenção prestada por meio da integração da rede pública de serviços de saúde e a formação de profissionais de saúde e da educação permanente através da incorporação e educação integral do processo saúde-doença-adoecimento, da promoção de saúde e do sistema de referência e contrarreferência ${ }^{5,14,15}$.

Nesta perspectiva, a formação por competência na atualidade apresenta uma importância essencial, já que esta facilita: a capacidade de afrontar demandas complexas, em um contexto determinado, pondo em relação e mobilizando pré-requisitos psicossociais que incluem aspectos tanto cognitivos como não cognitivos ${ }^{16}$; e demanda a integração de saberes essenciais que constituem o núcleo da competência, tendo como referência as formas de atuar mediante as quais se aplica o conhecimento e se resolvem os problemas tanto pessoais como profissionais nos diferentes cenários da sociedade do conhecimento ${ }^{17}$.

Assim, a formação do nutricionista deverá primar por um processo de ensino e aprendizagem capaz de colocar no mercado de trabalho profissionais que, além da competência técnica, estejam preparados para compreender, analisar e intervir nos problemas sociossanitários dos locais e cenários onde atuam, tendo como referência a formação cidadã e a busca da justiça social $^{9,17}$.

Nesta conjuntura, foi lançada em 2009 a $M a-$ triz de Ações de Alimentação e Nutrição na Aten- 
ção Básica de Saúde ${ }^{18}$ (MAANABS), elaborada pelo Observatório de Políticas de Segurança Alimentar e Nutrição da Universidade de Brasília (OPSAN/UnB), cujo objetivo principal é sistematizar e organizar as ações de alimentação e nutrição e o cuidado nutricional para integrarem o rol de ações de saúde desenvolvidas na atenção básica à saúde ${ }^{18}$.

Tendo em vista a importância de se avaliar a discussão da formação dos nutricionistas frente às necessidades impostas no cotidiano profissional no contexto do SUS, o presente estudo, de caráter bibliográfico, teve duplo objetivo: realizar uma revisão crítica dos estudos que orientam a formação e a prática dos nutricionistas no contexto da ABS no SUS, e avaliar a contribuição da (MAANABS) $)^{18}$ para esta discussão.

\section{Métodos}

Realizou-se uma revisão bibliográfica, tendo como pergunta norteadora: Como a matriz de ações de alimentação e nutrição na atenção básica de saúde pode contribuir para a formação do nutricionista no contexto da educação por competências no mundo contemporâneo?

\section{Critérios de inclusão e exclusão de estudos}

Foram levantados estudos publicados até fevereiro de 2013, nos idiomas português, inglês ou espanhol realizados no Brasil ou no exterior que abordassem a formação por competências dos nutricionistas e/ou a atuação destes na ABS no Brasil. Ou seja, os estudos que não envolviam o SUS, que tinham como foco outros níveis de atenção que não a primária, não envolviam a alimentação e nutrição, formação do nutricionista e publicações de outra natureza foram excluídos.

\section{Estratégia de busca e seleção de estudos}

Foi desenvolvido um protocolo para conduzir de forma sistematizada a revisão (Figura 1). Para a seleção dos estudos, foram consultadas as seguintes bases de dados em saúde:

Scientific Electronic Library Online Brasil (SciELO) e Biblioteca Virtual em Saúde (BVS, Ministério da Saúde, Brasil) que inclui como base dados - Ciências da Saúde em Geral: Lilacs, Ibecs, Medline, Biblioteca Cochrane; Portal de Evidências Revisões Sistemáticas, Ensaios Clínicos, Sumários de Evidência, Avaliações Econômicas em Saú- de, Avaliações de Tecnologias em Saúde, Diretrizes para Prática Clínica; Áreas Especializadas CidSaúde, Desastres, Hisa, Homeoindex, Leyes, Medcarib, Repidisca; Organismos Internacionais Paho, Wholis; Lis - Localizador de Informação em Saúde; DeCS - Terminologia em Saúde; Acesso a Documentos Scad - serviço de cópia de documentos, Catálogo de revistas científicas.

- Três conjuntos de intersecção de termos de busca bibliográfica foram combinados: Nutricionista [Nutritionist], Politica Nacional de Alimentação e Nutrição [PNAN]; Diretrizes Curriculares Nacionais na saúde, Educação baseada em competências; Atenção primária à saúde [atenção primária à saúde/cuidados primários de saúde/atenção básica à saúde (primary health care), saúde da família (family health), centros de saúde/unidades básicas de saúde (community health centers), serviços de saúde (health services)].

- Cada termo foi cruzado individualmente com outro, de forma que garantisse a inclusão de todos os estudos relacionados ao tema. Foram pesquisadas também as listas de referência dos estudos, a fim de identificar estudos relevantes não identificados na busca. Os nomes dos autores dos estudos considerados mais relevantes também foram utilizados nas buscas de outros estudos.

Como primeira avaliação, foram observados o título e o resumo dos artigos, sendo rejeitados aqueles que não atendiam aos critérios de inclusão ou apresentavam algum critério de exclusão. Nos casos de incerteza, o texto completo foi consultado para confirmar a elegibilidade do estudo.

De forma complementar, foram pesquisados documentos e publicações em portais do Sistema de Legislação em Saúde (Saúde-legis, Ministério da Saúde, Brasil) e Legislação em Vigilância Sanitária (e-legis, Agência Nacional de Vigilância Sanitária - Anvisa, Brasil).

\section{A Atenção Primaria à Saúde e a atuação do nutricionista}

O marco histórico da APS foi a Conferência Internacional Sobre Cuidados Primários de Saúde, realizada em Alma-Ata em 1978 ${ }^{19}$. "De acordo com a declaração de Alma Ata, a APS corresponde aos cuidados essenciais à saúde, baseados em tecnologias acessíveis, que levam os serviços de saúde o mais próximo possível dos lugares de vida e trabalho das pessoas, constituindo assim o primeiro nível de contato com o sistema nacional de saúde e o primeiro elemento de um processo contínuo de atenção. Desde então, diversos 




Critérios de exclusão
Estudos repetidos, que
não envolviam o SUS,
que tinham como foco
outros níveis de atenção
que não a primária, que
não envolviam a
alimentação e nutrição,
formação do
nutricionista e
publicações de outra
natureza.

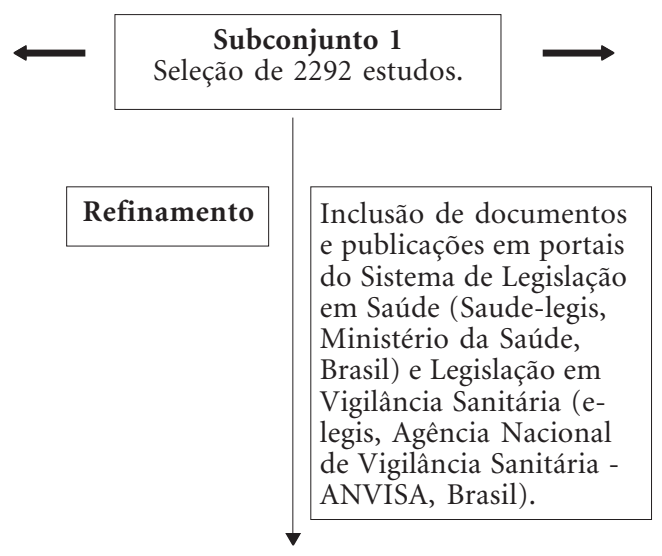

Critérios de inclusão Estudos publicados até fevereiro de 2013, nos idiomas português, inglês ou espanhol realizados no Brasil ou exterior que abordassem a formação por competências dos nutricionistas e/ou a atuação destes na atenção primária no Brasil.

Subconjunto 2

Seleção de 92 estudos para análise.

Figura 1. Protocolo de busca nas bases de dados científicas e critérios de seleção dos estudos para análise até Fevereiro de 2013.

autores vêm propondo definições sobre a APS, destacando-se os estudos da pesquisadora Bárbara Starfield, referência adotada pelo Ministério da Saúde do Brasil" ${ }^{20}$.

Segundo Starfield ${ }^{21}$, a APS forma a base e determina o trabalho de todos os outros níveis de atenção dos sistemas de saúde, promovendo a organização e a racionalização da utilização dos recursos, tanto primários como especializados, direcionados para a promoção, a manutenção e a melhoria da saúde. Assim, a APS é aquele nível do sistema de saúde que oferece a entrada do usuário, a porta, para todas as novas necessidades e problemas, fornecendo atenção sobre a 
pessoa (não direcionada para a enfermidade), no decorrer do tempo e para todas as condições.

O trabalho, nesse nível, baseia-se em uma nova concepção de saúde, não mais centrada somente na assistência à doença, mas, sobretudo, na promoção da qualidade de vida e na intervenção nos fatores que a colocam em risco, pela incorporação das ações programáticas de uma forma mais abrangente e do desenvolvimento de ações intersetoriais.

No contexto brasileiro, a expansão da atenção primária, impulsionada pelo processo de descentralização do SUS, além de operar mudanças na gestão e prestação de serviços no setor, também vem transformando o mercado de trabalho em saúde, sendo que a descentralização dessa força de trabalho constitui, atualmente, uma realidade. Nos dias atuais, a busca da integralidade é fundamental para a formação de cada profissional da área da saúde. Sendo assim, surge em 1994, no cenário brasileiro a Estratégia de Saúde da Família (ESF), reorientando o modelo assistencial, justificada pela necessidade de substituição do modelo historicamente centrado na doença e assistência médica individualizada, curativa e medicamentosa por um novo modelo sintonizado com os princípios do SUS, tais como universalidade, equidade, hierarquização, integralidade da atenção e participação social ${ }^{22}$.

Dentro deste contexto, o Brasil ainda é marcado pela dupla carga de doenças, com a convivência das infecciosas e transmissíveis, desnutrição e carências nutricionais específicas e de Doença Crônicas Não transmissíveis (DCNT) relacionadas à alimentação, tais como obesidade, hipertensão, doenças cardiovasculares, diabetes e alguns tipos de câncer, em todas as faixas de renda da população, em particular entre as famílias de menor poder socioeconômico ${ }^{23}$.

Compõem ainda esse quadro de insegurança alimentar e nutricional, presente entre parcela significativa das famílias brasileiras, as infecções e as doenças transmissíveis, quase sempre associadas a situações de pobreza e precariedade nas condições de alimentação e do espaço geográfico em que vivem. A eliminação da desnutrição infantil - atualmente expressa por déficits de crescimento - e das carências nutricionais por micronutrientes, como anemia e hipovitaminose A, ainda constituem desafios para os gestores do SUS e para todos os sujeitos envolvidos na atenção à saúde ${ }^{24}$.

Esses problemas de saúde e nutrição determinam uma mobilização de recursos econômicos, financeiros, políticos e sociais que poderiam ser evitados a partir de ações de promoção e prevenção, reduzindo também os custos no sistema de saúde en,26. $^{25}$.

Nesse sentido, a APS pode constituir uma medida de alta relevância para o desenvolvimento social de um país, quando atua de maneira oportuna e evita a referência de casos, ou mesmo a procura direta aos níveis de média e alta complexidade do sistema de saúde, sabidamente mais escassos e onerosos. Fortalecer e qualificar o cuidado nutricional no âmbito da atenção primária é uma forma mais econômica, ágil, sustentável e eficiente de prevenir a ocorrência de novos casos de obesidade e doenças associadas à má alimentação do que referenciá-los para o atendimento hospitalar, num futuro próximo, em decorrência de suas complicações ${ }^{27}$.

Desde esta perspectiva, salienta-se a importância estratégica da atuação do profissional nutricionista na APS, visto que este é o profissional capacitado para agir na resolução de problemas alimentares e nutricionais, e na promoção da saúde e prevenção de agravos e enfermidades relacionadas à insegurança alimentar.

No contexto da reorganização da atenção à saúde no âmbito do trabalho com grupos populacionais, comunidade e famílias, a abordagem de problemas relacionados com a alimentação e nutrição vem tornando-se necessária, visto o atual perfil de morbimortalidade da população brasileira, o qual indica elevação das DCNT como o diabetes mellitus, a obesidade, as neoplasias, a hipertensão arterial e as hiperlipidemias; que por sua vez estão diretamente relacionadas com a alimentação, a nutrição e o estilo de vida da população $0^{28,29}$.

Destaca-se, portanto, o importante papel do nutricionista na promoção de reeducação dos hábitos alimentares da população fazendo a prevenção de doenças e a promoção da saúde por meio do estimulo à mudança de estilo de vida e da incorporação de hábitos de vida saudáveis com consecutiva melhoria da qualidade de vida ${ }^{30}$.

Estas questões foram corroboradas nos estudos desenvolvidos por Santos ${ }^{30}$, nos quais médicos e enfermeiros salientaram as dificuldades que possuem para orientar seus pacientes sobre alimentação e relatam também que não têm formação adequada na área de nutrição, sendo esses os profissionais que dão orientações dietéticas quando não há um nutricionista na ESF. Assim, a inclusão do nutricionista na APS apresenta-se como uma necessidade político-social relevante.

Sua inserção apoia-se principalmente na aprovação da Política Nacional de Alimentação e 
Nutrição pelo Ministério da Saúde, a qual delega ao setor a realização de ações e formulação de políticas públicas voltadas à alimentação e nutrição, direcionadas à promoção de saúde, à prevenção e ao controle de deficiências nutricionais e DCNT ${ }^{31}$.

Nesse sentido, em janeiro de 2008 entrou em vigor o Núcleo de Apoio à Saúde da Família (NASF), criado pelo Ministério da Saúde através da portaria $\mathrm{GM} \mathrm{n}^{\circ} 154$, de 24 de janeiro de 2008, para melhorar a qualidade da APS através de equipes compostas por profissionais de diferentes áreas de conhecimento, dentre eles o nutricionista, contribuindo com a promoção de práticas alimentares saudáveis ${ }^{32}$.

O NASF vem pôr em prática o compromisso da integração de alimentação e nutrição com o setor saúde em relação aos componentes de Segurança Alimentar e Nutricional visando o direito humano à alimentação adequada, através da inserção do nutricionista na APS. Com a atuação do nutricionista, a comunidade local é beneficiada por um profissional capacitado para apoiar a realização de ações educativas sobre a alimentação e a nutrição. A competência do nutricionista para integrar a equipe ESF está estabelecida em sua formação acadêmica, a qual o instrumentaliza a realizar o diagnóstico nutricional da população, tornando-o, assim, o único profissional a receber uma instrução específica que lhe permite, a partir desse diagnóstico e da observação dos valores socioculturais, propor orientações dietéticas cabíveis e necessárias, adequando-as aos hábitos da unidade familiar, à cultura, às condições fisiológicas dos grupos e à disponibilidade de alimentos ${ }^{30}$.

Nos serviços de saúde, através das consultas com monitoramento do estado nutricional, há possibilidade de diagnósticos longitudinais, o que é de grande importância para a melhoria das condições alimentares e nutricionais da comunidade. Com a coleta de dados antropométricos integrados a outros marcadores de saúde como hipertensão e diabetes, há uma resposta mais efetiva em relação à necessidade de cada paciente ${ }^{33}$.

Trata-se, portanto, de um profissional apto a participar efetivamente da recriação das práticas de atenção à saúde no Brasil, nos moldes idealizados pelo movimento da reforma sanitária. Por este caminho, pode-se afirmar que uma assistência à saúde da família brasileira, cujo objetivo seja transformar a história das práticas e dos resultados das intervenções, não poderá prescindir da atuação do nutricionista.

Além das questões alimentares, o nutricionista em seu trabalho deve incentivar a produ- ção agrícola local, o que levará à geração de renda e empregos na região ${ }^{34}$.

Para atuar na saúde da família, o nutricionista necessita de uma formação voltada para questões sociais, com uma capacidade de leitura de problemas locais, como saneamento e habitação, em todo raio de abrangência, por exemplo, do NASF. Além de ser um especialista em alimentação humana, ele é um profissional de saúde coletiva. Como a interiorização desses serviços ainda deixa a desejar em todo o Brasil acredita-se que haja número insuficiente de nutricionistas em diversas áreas, nas quais a desnutrição e a má alimentação se manifestam de forma preocupante ${ }^{34}$.

Com vistas a todos esses benefícios da atuação do nutricionista junto à comunidade, é dever das universidades formar profissionais com este perfil sensibilizando os municípios para a necessidade de que o nutricionista integre suas equipes de saúde.

\section{As Diretrizes curriculares nacionais dos cursos de graduação em nutrição}

Nas DCN destaca-se a conformação diferenciada em relação ao extinto currículo mínimo. A ênfase não é mais em disciplinas, conteúdos e carga horária, mas no delineamento do perfil do egresso que cada universidade pretende formar e entregar à sociedade ao final da graduação, considerando suas necessidades loco regional ${ }^{35}$. Inclui elementos que visam subsidiar os estudantes para compreender e atuar diante das necessidades de saúde da população.

Dentre as inovações propostas, destacam-se: a) o estímulo à realização de atividades complementares (estágios, cursos, monitorias, projetos de extensão), que devem ocorrer ao longo do curso; b) flexibilização do regime de oferta das disciplinas (seriado semestral, seriado anual, créditos, módulos); c) incorporação de exigências para a gestão do curso, como a elaboração participativa do projeto pedagógico e d) direcionamento para uma distribuição equitativa da carga horária de estágio ( $20 \%$ do total) nas três principais áreas de atuação do nutricionista (nutrição clínica, nutrição social, administração de refeições coletivas) ${ }^{9}$.

As DCN também abordam as avaliações do desempenho dos alunos, que deverão incluir competências (conhecimentos, habilidades e atitudes), que incentivem a autoavaliação do próprio curso, em consonância "com o sistema de avaliação e a dinâmica curricular definidos pela Instituição de Ensino Superior (IES)"'13. 
As DCN apontam para o perfil do egresso do curso de nutrição que a universidade deve formar, reforçando a formação generalista e ampliando suas competências e espectro de atuação:

I- Nutricionista, com formação generalista, humanista e crítica, capacitado a atuar, visando à segurança alimentar e à atenção dietética, em todas as áreas do conhecimento em que alimentação e nutrição se apresentem fundamentais para a promoção, a manutenção e a recuperação da saúde e para a prevenção de doenças de indivíduos ou grupos populacionais, contribuindo para a melhoria da qualidade de vida, pautado em princípios éticos, com reflexão sobre a realidade econômica, política, social e cultural; II - Nutricionista com Licenciatura em Nutrição capacitado para atuar na Educação Básica e na Educação Profissional em Nutrição ${ }^{13}$.

Observa-se, por meio das DCN, que o conteúdo principal não deve centrar-se em matérias, disciplinas e carga horária, mas no delineamento do perfil profissional, dos princípios que devem reger a prática do nutricionista, na discriminação das competências requeridas, objetivando tornar o aluno mais capaz de lidar com as necessidades de saúde-doença da população e suas características culturais e regionais. A formação do nutricionista deve contemplar as necessidades sociais e de saúde, com ênfase no SUS ${ }^{13}$.

Um ponto convergente entre o currículo praticado desde 1975 e as DCN é a manutenção da orientação dos conteúdos de estudo, excetuando a delimitação dos ciclos profissional e básico ${ }^{7}$. Os conteúdos propostos pelas DCN são: ciências biológicas e da saúde, ciências sociais, humanas e econômicas, ciências da alimentação e nutrição, e ciência dos alimentos ${ }^{13}$. Ressalta-se, que esta convergência, expressa, na verdade, uma ambiguidade. Para Santos ${ }^{30}$, permanece o quadro teórico fragmentário, contrapondo o "preceito da aplicação de conhecimentos com visão holística do ser humano", defendido nas DCN.

Um aspecto positivo a ser destacado é o direcionamento para uma distribuição equitativa da carga horária de estágio nas três principais áreas de atuação do nutricionista, a saber: nutrição clínica, nutrição social e administração de refeições coletivas. Outras inovações que se observam são: a) orientação de atividades complementares, com ênfase de que devem ser conduzidas ao longo do curso; b) flexibilização do regime de oferta das disciplinas (seriado semestral, seriado anual, créditos, módulos); e, c) incorporação de exigências para a organização do curso, como a elaboração participativa de um projeto pedagógico ${ }^{7}$. Desta- ca-se ainda que a questão da segurança alimentar, embora não seja uma atribuição nova, aparece pela primeira vez explícita em parâmetro curricular oficial de Nutrição'

Assim, fica evidenciado, que as DCN representam, no momento, o que de mais atualizado existe em termos de orientação regulamentar para o curso de Nutrição em âmbito nacional. A seu favor cooperaram reflexões e sugestões da própria categoria, com participação das universidades.

As DCN, fruto de uma construção coletiva, marcam uma nova fase do currículo de Nutrição, constituem hoje um instrumento oficial de orientação a ser considerado na criação de cursos de Nutrição e avaliação/reformulação dos que já estão instituídos, como também do que se conseguiu produzir após longos anos de reflexão, debate e proposições. Por fim, considerando que as DCN introduzem a formação por competências no Brasil, a seguir será apresentado um recorte do debate sobre o conceito de competências que tem sido travado na área de Educação, com o intuito contextualizar a discussão.

\section{Formação por Competências}

O termo "competência” é polissêmico, complexo e controverso, e inicialmente se presta a múltiplas interpretações e matiz ${ }^{6}$. No Brasil, esta complexidade gerou e ainda gera muita discussão, porque a partir da entrada em vigência da LDB/1996 o ensino por competências passou a ser palavra de ordem ${ }^{36}$. Segundo as DCN, o sistema universitário brasileiro deve se centrar em competências, sejam estas gerais ou transversais e específicas ou técnicas.

A noção de competências é de tal forma polissêmica que poderíamos listar um conjunto de definições a elas conferidas. Sem embargo, Deluiz ${ }^{37}$ aponta três tipos de riscos na abordagem acrítica do conceito de competência. O primeiro seria a mera adequação da formação às necessidades da reestruturação econômica e às exigências empresariais. O segundo privilegia a sua "abordagem individualizada e individualizante", desconsiderando que as competências são construídas ao longo de um percurso, onde se sobressai a vivência coletiva. $O$ terceiro risco seria “... a preocupação com o produto (resultados) e não com o processo de construção das competências", sem questionar como estas se desenvolvem, transmitem e devem ser avaliadas.

Por sua vez, a definição de competências destacadas por Perrenoud ${ }^{38}$, que tem sido comparti- 
lhada por outros autores ${ }^{7,38,39}$, enfatiza: competência como "uma capacidade de agir eficazmente em um determinado tipo de situação, apoiada em conhecimentos, mas sem limitar-se a eles". A competência não é visível e se constrói a partir de situações que o indivíduo enfrenta com maior frequência. Já a habilidade seria um esquema com certa complexidade, existindo o estágio prático, que procede em geral de um treinamento intensivo.

Ao ser utilizada no âmbito do trabalho, essa noção torna-se plural, buscando designar os conteúdos particulares de cada função em uma organização de trabalho. A transferência desses conteúdos para a formação, orientada pelas competências que se pretende desenvolver nos educandos, dá origem ao que chamamos de 'pedagogia das competências', isto é, uma pedagogia definida por seus objetivos e validada pelas competências que produz ${ }^{38}$.

Esse deslocamento da qualificação para as competências no plano do trabalho produziu, no plano pedagógico, outro deslocamento, a saber: do ensino centrado em saberes disciplinares para um definido pela produção de competências verificáveis em situações e tarefas específicas e que visa a essa produção, que caracteriza a 'pedagogia das competências'.

Essas competências devem ser definidas com referência às situações que os alunos deverão ser capazes de compreender e dominar. A 'pedagogia das competências' passa a exigir, então, tanto no ensino geral quanto no profissionalizante, que as noções associadas (saber, saber-fazer) sejam acompanhadas de uma explicitação das atividades (ou tarefas) em que elas podem se materializar e se fazer compreender, explicitação esta que revela a impossibilidade de se dar uma definição a essas noções separadamente das tarefas nas quais elas se materializam ${ }^{40}$.

A 'pedagogia das competências' é caracterizada por uma concepção eminentemente pragmática, capaz de gerir as incertezas e levar em conta mudanças técnicas e de organização do trabalho às quais deve se ajustar ${ }^{41}$.

O MEC, por meio do Conselho Nacional de Educação (CNE), homologou as DCN com o entendimento de que um currículo baseado em competência parte da análise do processo de trabalho, da qual se constrói uma matriz referencial a ser transposta pedagogicamente para uma organização modular, adotando-se uma abordagem metodológica baseada em projetos ou resolução de problemas ${ }^{41}$.

O CNE define competência profissional como a capacidade de mobilizar, articular e colocar em ação valores, conhecimentos e habilidades necessários para o desempenho eficiente e eficaz de atividades requeridas pela natureza do trabalho ${ }^{7,9}$. Duas posições contrárias são identificadas entre os autores que analisam o encaminhamento dado pelo MEC. A primeira associa tal orientação curricular com o avanço da ideologia neoliberal, que buscaria subordinar a educação aos interesses de mercado ${ }^{42}$. A segunda correlaciona as DCN com a aprendizagem significativa, na perspectiva de uma nova pedagogia, que se opõe ao modelo clássico de ensino, de superação da valorização do conhecimento teórico em detrimento da prática $^{43}$. O texto das DCN permite, por suas ambiguidades, apropriação total ou parcial de ambas concepções ${ }^{7}$.

Além de atender o propósito de reordenar a relação entre escola/universidade e emprego, a 'pedagogia das competências' visa também a institucionalizar novas formas de educar os trabalhadores no contexto político-econômico neoliberal, entremeado a uma cultura chamada de pós-moderna. Por isto, a 'pedagogia das competências' não se limita à escola/universidade, mas visa a se instaurar nas diversas práticas sociais pelas quais as pessoas se educam. Nesse contexto, a noção de competência vem compor o conjunto de novos signos e significados talhados na cultura expressiva do estágio de acumulação flexível do capital, desempenhando um papel específico na representação dos processos de formação e de comportamento do trabalhador na sociedade ${ }^{38}$.

Assim, o desenvolvimento de uma pedagogia centrada nessa noção possui validade econômico-social e também cultural, posto que à educação se confere a função de adequar psicologicamente os trabalhadores aos novos padrões de produção. O novo senso comum, de caráter conservador e liberal, compreende que as relações de trabalho atuais e os mecanismos de inclusão social se pautam pela competência individual ${ }^{38}$.

Nesta perspectiva, a competência, inicialmente um aspecto de diferenciação individual, é tomada como fator econômico e se reverte em benefício do consenso social, envolvendo todos os trabalhadores supostamente numa única classe: a capitalista. Ao mesmo tempo, forma-se um consenso em torno do capitalismo como o único modo de produção capaz de manter o equilíbrio e a justiça social. Em síntese, a questão da luta de classe é resolvida pelo desenvolvimento e pelo aproveitamento adequado das competências individuais, de modo que a possibilidade de inclusão social subordina-se à capacidade de adaptação natural às relações contemporâneas. Assim, 
a flexibilidade econômica vem acompanhada da psicologização da questão social ${ }^{38}$.

A noção de competência situa-se, então, no plano de convergência entre a teoria integracionista da formação do indivíduo e da teoria funcionalista da estrutura social. A primeira demonstra que a competência torna-se uma característica psicológico-subjetiva de adaptação do trabalhador à vida contemporânea. A segunda situa a competência como fator de consenso necessário à manutenção do equilíbrio da estrutura social, na medida em que o funcionamento desta última ocorre muito mais por fragmentos do que por uma sequência de fatos previsíveis ${ }^{38}$.

O processo de construção do conhecimento pelo indivíduo, por sua vez, seria o próprio processo de adaptação ao meio material e social. Nesses termos, o conhecimento não resultaria de um esforço social e historicamente determinado de compreensão da realidade para, então, transformá-la, mas sim, das percepções e concepções subjetivas que os indivíduos extraem do seu mundo experiencial. O conhecimento ficaria limitado aos modelos viáveis de inteiração com o meio material e social, não tendo qualquer pretensão de ser reconhecido como representação da realidade objetiva ou como verdadeiro ${ }^{40}$.

A 'pedagogia das competências' reconfigura, então, o papel da escola/universidade. Se a escola moderna comprometeu-se com a sustentação do núcleo básico da socialização conferido pela família e com a construção de identidades individuais e sociais, contribuindo, assim, para a identificação dos projetos subjetivos com um projeto de sociedade; na pós-modernidade a escola é uma instituição mediadora da constituição da alteridade e de identidades autônomas e flexíveis, contribuindo para a elaboração dos projetos subjetivos no sentido de torná-los maleáveis o suficiente para se transformarem no projeto possível em face da instabilidade da vida contemporânea ${ }^{40}$.

Atuar na elaboração dos projetos possíveis é construir um novo profissionalismo que implica preparar os indivíduos para a mobilidade permanente entre diferentes ocupações numa mesma empresa, entre diferentes empresas, para o subemprego, para o trabalho autônomo ou para o não trabalho. Em outras palavras, a 'pedagogia das competências' pretende preparar os indivíduos para a adaptação permanente ao meio social instável da contemporaneidade ${ }^{41}$.

\section{Competência: um conceito polissêmico}

As competências englobam várias habilidades harmonicamente desenvolvidas. Elas norteiam a seleção de conteúdos, sua apropriação, comunicação, produção ou reconstrução. Assim, uma competência possibilita a mobilização de conhecimentos e esquemas para o enfrentamento de situações diversas. Seria a capacidade de encontrar recursos e respostas criativas e eficazes para problemas inéditos que se apresentam. Este alcance dependeria de uma formação calcada numa prática pedagógica contextualizada e interdisciplinar, onde o aluno desempenha um papel de sujeito, com a incorporação de "todos os fatores que fazem parte do ser unitário, ou seja, fatores afetivos, sociais e cognitivos" 39

De forma complementar, segundo Lizarraga $^{6}$ a competência faz referência a uma capacidade que se projeta na forma em que uma pessoa utiliza todos os seus recursos pessoais (habilidades, conhecimentos, atitudes e experiências) para resolver adequadamente uma tarefa em um contexto definido (...).

Por sua vez, Aretio et al. ${ }^{16}$ destacam que "educar não implica imprimir sem sentido uns conteúdos ou destrezas aprendidos, sem saber dotar-lhes de sentido e saber aplicá-los em cada situação de forma autônoma e responsável”. Nesta linha, vem cobrando uma importância muito singular a formação por competências ao entender que esta nos ajudará a compreender melhor este novo desenho de aprendizagem, ou seja, a demonstrada capacidade de utilizar conhecimentos, destrezas e habilidades pessoais, sociais e/ou metodológicas, em situações de estudo ou de trabalho e em desenvolvimento profissional e/ou pessoal para poder realizar-se e participar ativamente na sociedade, ser capaz de aprender ao longo de toda sua vida e estar preparada para as demandas em permanente mudanças que se apresentam na sociedade do conhecimento na qual vivemos. O que vai requerer um:

Saber - conhecimentos teóricos específicos de cada âmbito profissional ou de uma área acadêmica.

- Saber fazer - conjunto de habilidades e destrezas cognitivas, emocionais, sociais ou procedimentais que permitem aplicar o conhecimento que se possui.

- Saber conviver - atitudes pessoais e interpessoais, habilidades... que facilitma a convivência e o trabalho com os demais (trabalho em equipe). 
- Saber utilizar estrategicamente o conhecimento e aperfeiçoar-se, graças às competências metacognitivas.

- Saber Ser - ajustes de valores, princípios, crenças e atitudes profissionalmente válidas, modo de perceber-se e viver no mundo.

A escolha do trabalho com competências aporta muitas vantagens à educação superior, destacando-se: o fomento à transparência dos programas acadêmicos e enfatiza os resultados da aprendizagem; assume uma educação centrada nos estudantes, e em suas capacidades de aprender e em seus compromissos e responsabilidades, satisfaz as demandas de uma sociedade em constante câmbio e com novos desafios formativos, e busca maiores níveis de ocupação profissional e compromissos com a cidadania ${ }^{6}$.

Por fim, Epstein e Hunert apud Bollela e Machado $^{35}$ definem competência profissional a partir de uma ampla revisão de literatura como: "o uso habitual e criterioso da comunicação, do conhecimento, do raciocínio, da capacidade de integração de dados, habilidade técnica, emoções, capacidade reflexiva, e capacidade de se manter atualizado, que o nutricionista lança mão para servir as pessoas e as comunidades que dele necessitam".

\section{A Matriz de ações de alimentação e nutrição na APS}

A matriz de ações de alimentação e nutrição é proposta ao se considerar as atribuições e responsabilidades elencadas na Política Nacional de Alimentação e Nutrição (PNAN). A PNAN traz em sua concepção - e explicitamente em seu texto introdutório - que a "a alimentação e a nutrição constituem requisitos básicos para a promoção e a proteção da saúde, possibilitando a afirmação plena do potencial de crescimento e desenvolvimento humano, com qualidade de vida e cidadania" Tais atributos estão ainda consignados na Declaração Universal dos Direitos Humanos, promulgada em 1948, que foram reafirmados no Pacto Internacional sobre Direitos Econômicos, Sociais e Culturais (1966) e incorporados à legislação nacional em $1992^{31}$.

Desta forma, a PNAN estabelece atribuições para o Ministério da Saúde (gestor federal da PNAN) como: estabelecer normas e prestar cooperação técnica aos estados e municípios, voltadas à implementação desta Política, sistematizando, inclusive, medidas de prevenção e manejo de problemas de nutrição em escala individual, familiar e comunitária; criar mecanismos que vinculem a transferência de recursos às instâncias estadual e municipal ao desenvolvimento de um modelo de atenção à saúde; estimular e apoiar a realização de pesquisas estratégicas no contexto desta Política ${ }^{31}$.

Já os gestores municipais, foram incumbidos de responsabilidades como: promover a capacitação de recursos humanos para operacionalizar as atividades específicas na área de alimentação e nutrição; implantar o atendimento da clientela portadora de agravos nutricionais instalados, envolvendo: a assistência alimentar, o controle de doenças intercorrentes e a vigilância dos irmãos e contatos, garantindo a simultaneidade da execução de ações específicas de nutrição e de ações convencionais de saúde; uniformizar procedimentos relativos à avaliação de casos, à eleição de beneficiários, ao acompanhamento e recuperação de desnutridos, bem como à prevenção e manejo de doenças que interferem no estado nutricional; identificar e atender situações individuais e coletivas de risco nutricional; obter informações representativas do consumo alimentar ${ }^{31}$.

Destarte, ao analisar o propósito principal da matriz (MAANABS) que é de sistematizar e organizar as ações de alimentação e nutrição e do cuidado nutricional para integrarem o rol de ações de saúde desenvolvidas na APS seu objetivo contribui com o aperfeiçoamento da ação governamental, especificamente aquela sob responsabilidade e gestão da PNAN (Portaria no 710 , de 10 de junho de $1999^{44}$ ), da Política Nacional de Atenção Básica (Portaria n. ${ }^{\circ}$ 648, de 28 de março de $2006^{45}$ ) e de Promoção da Saúde (Portaria n. ${ }^{\circ}$ 687, de 30 de março de $2006^{46}$ ), a partir da adoção de ações de alimentação e nutrição na atenção básica em saúde, num esforço convergente e complementar às demais ações que já vêm sendo implementadas pelos diversos programas públicos de saúde ofertados, em especial a Estratégia Saúde da Família.

Na construção da matriz se considerou, previamente, alguns elementos e conceitos estratégicos e de cunho organizacional. Esses pressupostos são: sujeitos das ações (o indivíduo, a família e a comunidade); níveis de intervenção (gestão das ações de alimentação e nutrição e cuidado nutricional propriamente dito) - o cuidado nutricional engloba ações de diagnóstico, promoção da saúde, prevenção de doenças, tratamento/cuidado/assistência; caráter das ações (universais - aplicáveis a quaisquer fases do curso da vida e específicas - aplicáveis a determinada(s) fase(s) do curso da vida).

A matriz (MAANABS) elenca ações prioritárias e algumas delas dizem respeito ao conheci- 
mento técnico específico da formação do nutricionista, quais sejam, as relações entre o homem e o alimento. Muitas delas são relacionadas ao cuidado nutricional direcionado aos indivíduos, e tiveram como base as determinações legais para a atuação profissional e os princípios que regem o SUS.

De forma complementar, destacam-se outras atribuições a serem compartilhadas entre os membros da equipe, podendo o nutricionista assumir o papel de profissional-referência para o desenvolvimento das ações, tendo a responsabilidade de orientar a abordagem mais adequada, estabelecer protocolos de atenção em nutri- ção, de referência e contrarreferência, desde que sejam preservadas as suas atribuições privativas.

Há ainda outro grupo de atribuições, especialmente aquelas relacionadas à promoção da saúde e à prevenção de doenças, que são de responsabilidade do conjunto de profissionais de saúde.

Inúmeros protocolos e condutas já estabelecidas na rede SUS dão suporte para esse tipo de ação. Neste caso, a matriz procura abranger ações de nutrição que venham a integrar o rol das ações já desenvolvidas pelas equipes de saúde. Assim, a MAANABS elenca como referencial teórico documentos legais (Quadro 1) e técnicos de apoio (Quadro 2) para o desenvolvimento das ações.

Quadro 1. Documentos Legais de apoio para o desenvolvimento das ações de alimentação e nutrição.

\begin{tabular}{|c|c|c|c|}
\hline $\begin{array}{c}\text { Tipo de } \\
\text { documento }\end{array}$ & Número & Ano & Descrição \\
\hline Lei & 8.080 & 1990 & Lei Orgânica do SUS ${ }^{47}$. \\
\hline Lei & 11.346 & 2006 & Lei Orgânica de Segurança Alimentar e Nutricional ${ }^{48}$. \\
\hline Portaria & 710 & 1999 & Aprova a Política Nacional de Alimentação e Nutrição $0^{44}$. \\
\hline Portaria & 2.246 & 2004 & $\begin{array}{l}\text { Institui e divulga as orientações básicas para a implementação das } \\
\text { ações de vigilância alimentar e nutricional no âmbito das ações } \\
\text { básicas de saúde no Sistema Único de Saúde - SUS }{ }^{49} \text {. }\end{array}$ \\
\hline Portaria & 2.509 & 2004 & $\begin{array}{l}\text { Dispõe sobre as atribuições e normas para a oferta e o monitoramento } \\
\text { das ações de saúde relativas às condicionalidades das famílias } \\
\text { beneficiárias do Programa Bolsa Família }{ }^{50} \text {. }\end{array}$ \\
\hline Portaria & 2.608 & 2005 & $\begin{array}{l}\text { Define recursos financeiros da vigilância em saúde para incentivar a } \\
\text { estruturação de ações de vigilância e prevenção de doenças e de } \\
\text { agravos não-transmissíveis por parte das Secretarias Estaduais e } \\
\text { Municipais de Saúde das capitais brasileiras }{ }^{51} \text {. }\end{array}$ \\
\hline Portaria & 399 & 2006 & $\begin{array}{l}\text { Contempla o Pacto firmado entre os gestores do SUS, em suas três } \\
\text { dimensões: pela Vida, em Defesa do SUS e de Gestão }{ }^{52} \text {. }\end{array}$ \\
\hline Portaria & 648 & 2006 & Aprova a Política Nacional de Atenção Básica ${ }^{45}$. \\
\hline Portaria & 1.010 & 2006 & $\begin{array}{l}\text { Institui as diretrizes para a promoção da alimentação saudável nas } \\
\text { escolas }^{53} \text {. }\end{array}$ \\
\hline Portaria & 687 & 2006 & Aprova a Política Nacional de Promoção da Saúde ${ }^{46}$. \\
\hline Portaria & 1.097 & 2006 & $\begin{array}{l}\text { Dispõe sobre as Diretrizes para a Programação Pactuada e Integrada } \\
\text { da Assistência em Saúde }{ }^{54} \text {. }\end{array}$ \\
\hline Portaria & 154 & 2008 & Cria os Núcleos de Apoio à Saúde da Família ${ }^{32}$. \\
\hline
\end{tabular}


Quadro 2. Documentos Técnicos de apoio para o desenvolvimento das ações de alimentação e nutrição.

\begin{tabular}{|c|c|c|c|}
\hline $\begin{array}{c}\text { Tipo de } \\
\text { documento }\end{array}$ & Órgão executor & Ano & Nome \\
\hline Manual $^{55}$ & $\begin{array}{l}\text { Ministério da Saúde. Secretaria de } \\
\text { Atenção à Saúde. Coordenação Geral da } \\
\text { Política de Alimentação e Nutrição. }\end{array}$ & 2005 & $\begin{array}{l}\text { Manual de atendimento da criança } \\
\text { com desnutrição grave em nível } \\
\text { hospitalar. }\end{array}$ \\
\hline $\begin{array}{l}\text { Guia } \\
\text { alimentar }\end{array}$ & $\begin{array}{l}\text { Ministério da Saúde. Secretaria de } \\
\text { Política de Saúde. Organização Pan } \\
\text { Americana da Saúde. }\end{array}$ & 2002 & $\begin{array}{l}\text { Guia alimentar para crianças menores } \\
\text { de dois anos. }\end{array}$ \\
\hline $\begin{array}{l}\text { Guia } \\
\text { alimentar }^{57}\end{array}$ & $\begin{array}{l}\text { Ministério da Saúde. Secretaria de } \\
\text { Atenção à Saúde. Departamento de } \\
\text { Atenção Básica. Coordenação-Geral da } \\
\text { Política de Alimentação e Nutrição. }\end{array}$ & 2006 & $\begin{array}{l}\text { Guia Alimentar para a População } \\
\text { Brasileira: Promovendo a Alimentação } \\
\text { Saudável. }\end{array}$ \\
\hline Cadernos $^{58}$ & $\begin{array}{l}\text { Brasil. Ministério da Saúde. Secretaria } \\
\text { de Atenção à Saúde. Departamento de } \\
\text { Atenção Básica. }\end{array}$ & 2006 & Obesidade. \\
\hline $\begin{array}{l}\text { Normas e } \\
\text { Manuais } \\
\text { Técnicos }\end{array}$ & Ministério da Saúde. Unicef. & 2007 & Carências de Micronutrientes. \\
\hline $\begin{array}{l}\text { Normas e } \\
\text { Manuais } \\
\text { Técnicos }^{60}\end{array}$ & $\begin{array}{l}\text { Ministério da Saúde. Secretaria de } \\
\text { Atenção à Saúde. Departamento de } \\
\text { Atenção Básica. }\end{array}$ & 2007 & $\begin{array}{l}\text { Alimentação e nutrição para as famílias } \\
\text { do Programa Bolsa Família: manual } \\
\text { para os agentes comunitários de saúde. }\end{array}$ \\
\hline Manual $^{61}$ & $\begin{array}{l}\text { Ministério da Saúde. Secretaria de } \\
\text { Atenção à Saúde. Departamento de } \\
\text { Atenção Básica. Coordenação-Geral de } \\
\text { Política de Alimentação e Nutrição. }\end{array}$ & 2005 & $\begin{array}{l}\text { Manual de Orientações sobre o Bolsa } \\
\text { Família. }\end{array}$ \\
\hline $\begin{array}{l}\text { Comunicação } \\
\text { e Educação } \\
\text { em saúde }^{62}\end{array}$ & $\begin{array}{l}\text { Ministério da Saúde. Secretaria de } \\
\text { Políticas de Saúde. Coordenação-Geral } \\
\text { da Política de Alimentação e Nutrição. }\end{array}$ & 2002 & Alimentos regionais brasileiros. \\
\hline Manual $^{63}$ & $\begin{array}{l}\text { Ministério da Saúde. Secretaria de } \\
\text { Atenção à Saúde. Departamento de } \\
\text { Atenção Básica. }\end{array}$ & 2008 & $\begin{array}{l}\text { Manual Técnico e Operacional do Pró- } \\
\text { Iodo. }\end{array}$ \\
\hline $\begin{array}{l}\text { Normas e } \\
\text { Manuais } \\
\text { Técnicos }^{64}\end{array}$ & Ministério da Saúde. & 2004 & $\begin{array}{l}\text { Sisvan: orientações básicas para a coleta, } \\
\text { processamento, análise de dados e } \\
\text { informação em serviços de saúde. }\end{array}$ \\
\hline Guia $^{65}$ & $\begin{array}{l}\text { Ministério da Saúde. Organização Pan- } \\
\text { Americana de Saúde. Coordenação- } \\
\text { Geral da Politica de Alimentação e } \\
\text { Nutrição. }\end{array}$ & 2002 & $\begin{array}{l}\text { Dez passos da Alimentação Saudável } \\
\text { para crianças menores de } 2 \text { anos. }\end{array}$ \\
\hline $\begin{array}{l}\text { Normas e } \\
\text { Manuais } \\
\text { Técnicos }{ }^{66}\end{array}$ & $\begin{array}{l}\text { Ministério da Saúde. Secretaria de } \\
\text { Atenção à Saúde. Departamento de } \\
\text { Atenção Básica. }\end{array}$ & 2004 & $\begin{array}{l}\text { Vitamina A Mais: Programa Nacional } \\
\text { de Suplementação de Vitamina A: } \\
\text { Condutas Gerais. }\end{array}$ \\
\hline Guia $^{67}$ & $\begin{array}{l}\text { Ministério da Saúde. Secretaria de } \\
\text { Vigilância em Saúde. Secretaria de } \\
\text { Atenção à Saúde. }\end{array}$ & 2004 & $\begin{array}{l}\text { Guia prático de preparo de alimentos } \\
\text { para crianças menores de } 12 \text { meses que } \\
\text { não podem ser amamentadas. }\end{array}$ \\
\hline $\begin{array}{l}\text { Normas e } \\
\text { Manuais } \\
\text { Técnicos }^{68}\end{array}$ & $\begin{array}{l}\text { Ministério da Saúde. Secretaria de } \\
\text { Vigilância em Saúde. Programa } \\
\text { Nacional de DST/AIDS. }\end{array}$ & 2006 & $\begin{array}{l}\text { Manual clínico de alimentação e } \\
\text { nutrição na assistência a adultos } \\
\text { infectados pelo HIV. }\end{array}$ \\
\hline $\begin{array}{l}\text { Comunicação } \\
\text { e Educação } \\
\text { em Saúde }\end{array}$ & $\begin{array}{l}\text { Ministério da Saúde. Secretaria de } \\
\text { Vigilância em Saúde. Programa } \\
\text { Nacional de DST/Aids. }\end{array}$ & 2006 & $\begin{array}{l}\text { Alimentação e nutrição para pessoas } \\
\text { que vivem com HIV e Aids. }\end{array}$ \\
\hline
\end{tabular}


Ao dar mais visibilidade às ações de alimentação e nutrição na ABS e fomentar a sua adequada gestão e execução no âmbito dos municípios brasileiros, a MAANABS trilha os caminhos que o SUS vem percorrendo, nesses últimos 20 anos, para municipalizar suas ações e garantir o direito à saúde a toda a população. Pensar na inserção do Nutricionista na ABS é investir em mudanças de realidades, que ocorram de modo permanente desde a graduação até o investimento em Educação Permanente.

Assim, é função da academia dotar a sociedade de profissionais capazes de transformar e contribuir com as políticas públicas, direcionando-as à promoção da saúde da população, mostrando que estas não devam ser de exclusividade do Estado, mas do resultado de pactuação entre setores, empoderamento da sociedade organizada, da distribuição de direitos e deveres entre o Estado e a Sociedade, entre indivíduos e coletivos e entre público e privado.
Desta forma, a matriz de ações contribui para a formação do nutricionista dentro do contexto da educação por competências ao servir de referencial de ações, competências e habilidades ao se formar indivíduos mais capazes de lidar com as necessidades do processo saúde-doença da população e suas características culturais e regionais com ênfase no SUS.

Espera-se também que ao utilizar a Matriz como referencial de formação, contribuir com o aperfeiçoamento desta e das ações governamentais, especificamente aquelas sob responsabilidade e gestão da Política Nacional de Alimentação e Nutrição (PNAN), da Política Nacional de Atenção Básica e de Promoção da Saúde, a partir da adoção de ações de alimentação e nutrição na atenção primária em saúde, num esforço convergente e complementar às demais ações que já vêm sendo implementadas pelos diversos programas públicos de saúde ofertados, em especial a Estratégia Saúde da Família.

\section{Colaboradores}

TS Junqueira trabalhou na pesquisa, na metodologia, na concepção e na redação final do artigo; e RMM Cotta na concepção, na correção e na redação final do artigo.

\section{Agradecimentos}

O presente trabalho foi realizado com o apoio da Coordenação de Aperfeiçoamento de Pessoal de Nível Superior (CAPES). 


\section{Referências}

1. Siqueira-Batista R, Gomes AP, Albuquerque VS, Cavalcanti FOL, Cotta RMM. Educação e competências para o SUS: é possível pensar alternativas à(s) lógica(s) do capitalismo tardio? Cien Saude Colet 2013; 18(1):159-170.

2. Capra F. Alfabetização ecológica. São Paulo: Cultrix; 2006.

3. Mendes EV. Uma agenda para a saúde. 2a ed. São Paulo: Hucitec; 2006.

4. Cotta RMM, Azeredo CM, Schott M, Martins PC, Franceschini SCC, Priore SE. Sobre o conhecimento e a consciência sanitária brasileira: o papel estratégico dos profissionais e usuários no sistema sanitário. Rev Med Minas Gerais 2006; 16(1):2-8.

5. Cotta RMM, Mendonça ET, Costa GD. Portfólios reflexivos: construindo competências para o trabalho no Sistema Único de Saúde. Rev Panam Salud Publica 2011; 30(5):415-421.

6. Lizarraga MLSDA. Competencias cognitivas en educacion superior. Madrid: Universitaria; 2010.

7. Soares NT. Diretrizes curriculares nacionais para cursos de graduação em nutrição e competências profissionais para atuação em segurança alimentar e nutricional: perspectivas de docentes do Ceará [dissertação]. Rio de Janeiro: Universidade do Estado do Rio de Janeiro; 2007.

8. Costa NSC. A formação do nutricionista, educação e contradição. 2a ed. Goiânia: UFG; 2002.

9. Soares NT, Aguiar ACD. Diretrizes curriculares nacionais para os cursos de nutrição: avanços, lacunas, ambiguidades e perspectivas. Revista de Nutrição 2010; 23(5):895-905.

10. Feuerwerker LCM. Gestão dos processos de mudança na graduação em Medicina. In: Marins JJN, organizador. Educação Médica em Transformação: instrumento para a construção de novas realidades. São Paulo: Hucitec, ABEM; 2004. p. 17-39

11. Fullan M. O significado da mudança educacional. Porto Alegre: Artmed; 2009.

12. Pasquim EM. Nutrição no Programa de Saúde da Família: Conhecimentos e Obstáculos entre Médicos, Enfermeiros e Agentes Comunitários de Saúde de Duas Cidades do Distrito Federal. Nutrição em Pauta 2002; 55:12-21.

13. Brasil. Resolução CNE/CES 5, de 7 de novembro de 2001. Institui diretrizes curriculares nacionais do curso de graduação em nutrição. Diário Oficial da União 2001; 9 nov.

14. Brasil. Programa Nacional de Reorientação da Formação Profissional em Saúde - Pró-saúde educação. Brasília: MDSMD; 2007.

15. Ribeiro AG, Ribeiro SMR, Dias CMGC, Ribeiro AQ, Castro FAF, Suárez-Varela MM, Cotta RM. Non-pharmacological treatment of hypertension in primary health care: A comparative clinical trial of two education strategies in health and nutrition. BMC Public Health 2011; 11:637.

16. Aretio LG, Cordella MR, Blanco MG. Claves para la educación - actores, agentes y escenarios en La sociedad actual. Madrid: Narcea AS Ediciones; 2009.
17. Cotta RMM, Silva LSD, Lopes LL, Gomes KDO, Cotta FM, Lugarinho R, Mitre SM. Construção de portfólios coletivos em currículos tradicionais: uma proposta inovadora de ensino-aprendizagem. Cien Saude Colet 2012; 17(3):787-796.

18. Brasil. Ministério da Saúde (MS). Matriz de ações de alimentação e nutrição na atenção básica de saúde. Brasília: MS; 2009.

19. Declaração de Alma-Ata. In: Conferência Internacional sobre Cuidados Primários de Saúde. Alma Ata, Cazaquistão; 6-12; 1978.

20. Gomes KO, Cotta RMM, Araújo RMA, Cherchiglia ML, Martins TCP. Atenção Primária à Saúde - a 'menina dos olhos' do SUS: sobre as representações sociais dos protagonistas do Sistema Único de Saúde. Cien Saude Colet 2011; 16(Supl. 1):881-892.

21. Starfield B. Atenção primária: equilíbrio entre necessidades de saúde, serviços e tecnologia. Brasília: UNESCO, Ministério da Saúde; 2002. 726p. [documento da Internet] 2008 [acessado 2008 jul 16]; [726 p.]. Disponível em: http://www.unesco.org.br/publicacoes/livros/atencaoprimaria/mostra_documento.

22. Gomes KO, Cotta RMM, Cherchiglia ML, Mitre SM, Batista RS. A Práxis do Agente Comunitário de Saúde no Contexto do Programa Saúde da Família: reflexões estratégicas. Saúde Soc 2009; 18(4):744-755,

23. Sistema Conselhos Federal e Regionais de Nutricionistas (SCFRN). O papel do nutricionista na atenção primária à saúde. Brasília: SCFRN; 2008.

24. Brasil. Ministério da Saúde (MS). Pesquisa nacional de demografia e saúde da criança e da mulher. PNDS 2006 - resultados preliminares. Brasília: MS; 2006.

25. World Cancer Researcher Fund (WCRF). American Institute For Cancer Research (AICR). Food, nutrition, physical activity, and the prevention of Cancer: a global perspective. Washington: AICR; 2007.

26. Sichieri R, Nascimento S. O Custo da obesidade para o Sistema Único de Saúde. Perspectivas para as próximas décadas. Taddei JA, coordenador. Jornadas Científicas do Núcleo Interdepartamental de Segurança Alimentar e Nutricional. Barueri: Minha Editora; 2007.

27. Dietitians of Canada. The role of the registered dietitian in primary health care: a national perspective. Canada, May, 2001. [Internet]. [acessado $2014 \mathrm{abr}$ 2]. Disponível em: http://www.dietitians.ca/Downloadable-Content/Public/phc-position-paper.aspx

28. Ribeiro AB, Cardoso MA. Construção de um questionário de freqüência alimentar como subsídio para programas de prevenção de doenças crônicas não transmissíveis. Rev Nutr 2002; 15(02):239-245.

29. Assis AMO, Santos SMC, Freitas MCS, Santos JM, Silva MCM. O Programa Saúde da Família: contribuições para uma reflexão sobre a inserção do nutricionista na equipe multidisciplinar. Rev Nutr 2002; 15(03):255-266.

30. Santos AC. A inserção do nutricionista na estratégia da saúde da família: o olhar de diferentes trabalhadores da saúde. Fam. Saúde Desenv 2005; 7(3):257265. 
31. Brasil. Ministério da Saúde (MS). Política Nacional de Alimentação e Nutrição. 2a ed. rev. Brasília: MS; 2003.

32. Brasil. Ministério da Saúde (MS). Portaria $n^{\circ} 154$, de 24 de janeiro de 2008. Cria os Núcleos de Apoio à Saúde da Família - NASF. Diário Oficial da União 2008; 25 jan.

33. Machado NMV, Viteritte PL, Goulart DAS, Pinheiro ARO. Reflexões sobre saúde, nutrição e a estratégia de saúde da família. 2006. [acessado 2008 abr 17]; [cerca de 2 p.]. Disponível em: http://dtr2004. saude.gov.br/nutricao/documentos/noticia_01_ 09_06.pdf

34. Silva NF. O Nutricionista na atenção básica. Conselho Federal de Nutrição. 2008. [acessado 2011 nov 15]; 51(1): [cerca de 3 p.]. Disponível em: http:/ /www.cfn.org.br/eficiente/repositorio/Artigos/ 51.pdf.

35. Bollela VB, Machado JLM. Internato baseado em competências - "Bridging the gaps". São Paulo: MedVance; 2010.

36. Moura e Silva ZAP. Falando de competências e Habilidades. APASE, Suplemento pedagógico. 2002. [acessado 2008 nov 25]; [cerca de 4 p.]. Disponível em: http://www.apase.hpg.ig.com.br/separata_10_ 2002_0002.htmp.

37. Deluiz N. A Globalização Econômica e os Desafios à Formação Profissional. Boletim do Senac 2004; 30(3):1.

38. Perrenoud P. Construir as Competências Desde a Escola. Porto Alegre: Artemed; 1999.

39. Ramos MN. Qualificação, competências e certificação: visão educacional. Brasília: Ministério da Saúde; 2001.

40. Guedes GG. As habilidades e o pensamento sistêmico: fatores decisivos na formação do administrador. Global Manager 2003; (5):45-59.

41. Ramos MN. Pedagogia das competências. Rio de Janeiro: Dicionário Fiocruz; 2008.

42. Ramos MN. A educação profissional pela pedagogia das competências: para além da superfície dos documentos oficiais. Educ. Soc. Campinas 2002; 23(80):401-422.

43. Maués OCW, Calixte E, Gauthier C. Duas perspectives diferentes em relação à abordagem por competências no ensino: os casos do Brasil e do Quebec. 25a Reunião Anual da Anped. Caxambu: ANPED; 2002.

44. Brasil. Portaria no 710 , de 10 de junho de 1999. Diário Oficial da União 1999; 11 jul.

45. Brasil. Portaria n. ${ }^{\circ}$ 648, de 28 de março de 2006. Aprova a Política Nacional de Atenção Básica, estabelecendo a revisão de diretrizes e normas para a organização da Atenção Básica para o Programa Saúde da Família (PSF) e o Programa Agentes Comunitários de Saúde (PACS). Diário Oficial da União 2006; 29 mar.

46. Brasil. Portaria n. ${ }^{\circ}$ 687, de 30 de março de 2006. Aprova a Política de Promoção da Saúde. Diário Oficial da União 2006; 31 mar.
47. Brasil. Presidência da República. Lei no 8.080, de 19 de setembro de 1990. Dispõe sobre as condições para a promoção, proteção e recuperação da saúde, a organização e o funcionamento dos serviços correspondentes e dá outras providências. Diário Oficial da União 1990; 19 set.

48. Brasil. Ministério da Saúde (MS). Lei no 11.346, de 15 de setembro de 2006. Cria o Sistema Nacional de Segurança Alimentar e Nutricional - SISAN com vistas em assegurar o direito humano à alimentação adequada e dá outras providências. Diário Oficial da União 2006; 18 set.

49. Brasil. Ministério da Saúde (MS). Portaria no 2.246, de 18 de outubro de 2004. Institui e divulga orientações básicas para a implementação das Ações de Vigilância Alimentar e Nutricional, no âmbito das ações básicas de saúde do Sistema Único de Saúde - SUS, em todo o território nacional. Diário Oficial da União 2004; 19 out.

50. Brasil. Ministério da Saúde (MS). Portaria no 2.509, de 18 de novembro de 2004. Dispõe sobre as atribuições e normas para a oferta e o monitoramento das ações de saúde relativas às condicionalidades das famílias beneficiárias do Programa Bolsa Família. Diário Oficial da União 2004; 19 nov.

51. Brasil. Ministério da Saúde (MS). Portaria no 2.608, de 31 de outubro de 2005. Define Municípios com adesão ao Programa Saúde na Escola de 2013 e os habilita ao recebimento de $20 \%$ (vinte por cento) do teto de recursos financeiros pactuados em Termo de Compromisso e dá outras providências. Diário Oficial da União 2005; 1 nov.

52. Brasil. Ministério da Saúde (MS). Portaria no 399, de 22 de fevereiro de 2006. Divulga o pacto pela saúde 2006 - consolidação do SUS e aprova as diretrizes operacionais do referido pacto. Diário Oficial da União 2006; 23 fev.

53. Brasil. Ministério da Saúde (MS). Portaria no 1.010 , de 8 de maio de 2006. Institui as diretrizes para a promoção da alimentação saudável nas escolas. Diário Oficial da União 2006; 9 maio.

54. Brasil. Ministério da Saúde (MS). Portaria no 1.097, de 22 de maio de 2006. Define o processo da programação pactuada e integrada da assistência em saúde seja um processo instituído no âmbito do sistema único de saúde. Diário Oficial da União 2006; 23 maio.

55. Brasil. Ministério da Saúde (MS). Secretaria de Atenção à Saúde. Coordenação Geral da Política de Alimentação e Nutrição. Manual de atendimento da criança com desnutrição grave em nível hospitalar. Brasília: MS; 2005. (Série A. Normas e Manuais Técnicos).

56. Brasil. Ministério da Saúde (MS). Secretaria de Política de Saúde. Organização Pan Americana da Saúde. Guia alimentar para crianças menores de dois anos. Brasília: MS, Organização Pan Americana da Saúde; 2002. (Série A. Normas e Manuais Técnicos; n. 107) 
57. Brasil. Ministério da Saúde (MS). Secretaria de Atenção à Saúde. Guia alimentar para a população brasileira: promovendo a alimentação saudável. Brasília: MS; 2008. (Série A. Normas e Manuais Técnicos)

58. Brasil. Ministério da Saúde (MS). Secretaria de Atenção à Saúde. Departamento de Atenção Básica. Obesidade. Brasília: MS; 2006. (Cadernos de Atenção Básica, n. 12) (Série A. Normas e Manuais Técnicos)

59. Brasil. Ministério da Saúde (MS), Unicef. Cadernos de Atenção Básica: Carências de Micronutrientes. Brasília: MS, Unicef; 2007. (Série A. Normas e Manuais Técnicos)

60. Brasil. Ministério da Saúde (MS). Secretaria de Atenção em Saúde. Departamento de Atenção Básica. Alimentação e nutrição para as famílias do programa bolsa família: manual para os agentes comunitários de saúde. Brasília: MS; 2010. (Série A. Normas e Manuais Técnicos)

61. Brasil. Ministério da Saúde (MS). Secretaria de Atenção à Saúde. Departamento de Atenção Básica. Coordenação-Geral de Política de Alimentação e Nutrição. Manual de Orientações sobre o Bolsa Família. 1a ed. Brasília: MS; 2005. (Série A. Normas e Manuais Técnicos)

62. Brasil. Ministério da Saúde (MS). Secretaria de Políticas de Saúde. Coordenação-Geral da Política de Alimentação e Nutrição. Alimentos regionais brasileiros. $1^{\text {a }}$ ed. Brasília: MS; 2002. (Série F. Comunicação e Educação em Saúde; n. 21)

63. Brasil. Ministério da Saúde (MS). Secretaria de Atenção à Saúde. Departamento de Atenção Básica. Manual Técnico e Operacional do Pró-Iodo: Programa Nacional para a Prevenção e Controle dos Distúrbios por Deficiência de Iodo. Brasília: MS; 2007. (Série A. Normas e Manuais Técnicos)
64. Brasil. Ministério da Saúde (MS). Vigilância alimentar e nutricional. Sisvan: orientações básicas para a coleta, processamento, análise de dados e informação em serviços de saúde. Brasília: MS; 2004. (Série A. Normas e Manuais Técnicos)

65. Brasil. Ministério da Saúde (MS). Dez passos para uma alimentação saudável: guia alimentar para crianças menores de 2 anos. Brasília: MS; 2003. 20 p.: il. (Série A. Normas e Manuais Técnicos)

66. Brasil. Ministério da Saúde (MS). Secretaria de Atenção à Saúde. Departamento de Atenção Básica. Manual de condutas gerais do Programa Nacional de Suplementação de Vitamina A. Brasília: MS; 2013.

67. Brasil. Ministério da Saúde (MS). Guia prático de preparo de alimentos para crianças menores de 12 meses que não podem ser amamentadas. Brasília: MS; 2004.

68. Brasil. Ministério da Saúde (MS). Secretaria de Vigilância em Saúde. Programa Nacional de DST/ AIDS. Manual clínico de alimentação e nutrição na assistência a adultos infectados pelo HIV. Brasília: MS; 2006. (Série A. Normas e Manuais Técnicos)

69. Brasil. Ministério da Saúde (MS). Secretaria de Vigilância em Saúde. Programa Nacional de DST/ Aids. Alimentação e nutrição para pessoas que vivem com HIV e Aids. Brasília: MS; 2006. (Série F. Comunicação e Educação em Saúde)

Artigo apresentado em 16/07/2013

Aprovado em 10/10/2013

Versão final apresentada em 15/10/2013 